\title{
Wie führende Unternehmen ihre Finanzfunktion weiterentwickeln
}

Strategische Aufgaben werden für die Finanzfunktion in Unternehmen immer wichtiger. Um diesen Aufgaben gerecht zu werden, müssen qualifizierte Mitarbeiter an das Unternehmen gebunden werden. Dies bestätigt auch eine aktuelle Studie der Beratungsgesellschaft PwC. Für die Studie wurden 200 international agierende Unternehmen untersucht. Dabei zeigt sich, dass die Möglichkeiten der Finanzfunktion zur Steuerung des Geschäftes oft noch nicht genügend genutzt werden. Fast jeder zweite Finanzchef empfindet die Qualität der Prognosen als nicht ausreichend. Laut $\mathrm{PwC}$ wird ein großes Potenzial, Informationen für Planung und Vorausschau besser aufzubereiten, bisher nicht ausgeschöpft.

Bei den Kosten für die Finanzfunktion zeigen sich deutliche Unterschiede zwischen den befragten Teilnehmern. Bei durchschnittlich aufgestellten Unternehmen sind die Kosten laut der Studie um 60 Prozent höher als bei Top-Unternehmen. Um Kosten zu sparen, lagern viele Unternehmen einen Teil des Rechnungswesens aus oder nutzen Shared Service Center. Auch neue Technologien kommen zum Einsatz - und das sehr erfolgreich. Der Zeitaufwand für das Budget Reporting soll laut PwC im Vergleich zu 2012 um 14 Prozent gesunken sein.

Mehr zum Thema:

Vogel, J./Wenk, O.: Reifegradmodelle in der Finanzabteilung von Unternehmen, in: Business + Innovation, Jg. 3 (2012), Heft 4, S. $16-23$,

SfP $^{*}$ www.springerprofessional.de/3560508

Sylvia Meier, Wiesbaden

\section{Deutscher Mittelstand boomt und beklagt Umsatzeinbußen durch Fachkräftemangel}

Mehr Umsatz, mehr Investitionen, steigende Beschäftigung, das sind die zentralen Ergebnisse des aktuellen Mittelstandsbarometers der Prüfungs- und Beratungsgesellschaft Ernst \& Young (EY). Die Studie wird halbjährlich durchgeführt. Ihr liegt eine Umfrage unter 3.000 mittelständischen Unternehmen im Dezember 2013 und Januar 2014 zugrunde.

55 Prozent der befragten Unternehmen rechnen demnach für 2014 mit steigenden Umsätzen von durchschnittlich 1,2 Prozent. 28 Prozent der Unternehmen planen deshalb, zusätzliche Mitarbeiter einzustellen. Das ist allerdings nicht so einfach. Mehr als sieben von zehn Mittelständlern bezeichnen es der Studie zufolge als schwierig, geeignete neue Mitarbeiter zu finden. Und sechs von zehn Unternehmen haben aktuell offene Stellen, die sie mangels Bewerber nicht besetzen können.
Umsatzeinbußen in Milliardenhöhe sind die Folge. So können Aufträge nicht angenommen werden, oder es wird weniger produziert, als abgesetzt werden könnte. Immerhin jedes neunte Unternehmen, so die Studie, beklagt Umsatzeinbußen von mehr als fünf Prozent aufgrund fehlender Fachkräfte. Weitere 42 Prozent der Unternehmen rechnen mit geringfügigen Umsatzeinbußen. Nach einer Analyse von EY summieren sich die Umsatzausfälle im deutschen Mittelstand damit auf hochgerechnet 31 Milliarden Euro jährlich.

Mehr zum Thema:

„Highpotentials sind stark umkämpft“, Online-Interview mit Stefan Rippler, 04.06.2013,

SSTID $^{*}$ www.springerprofessional.de/4445328

Vera Treitschke, Wiesbaden 